\title{
À CHACUN SON PSY. LA DIFFUSION DES PRATIQUES PSYCHOLOGIQUES EN PRISON
}

\section{Corinne Rostaing}

Presses de Sciences Po | «Sociologies pratiques »

2008/2 $\mathrm{n}^{\circ} 17$ | pages 81 à 94

ISSN 1295-9278

ISBN 2130569312

\section{Article disponible en ligne à l'adresse :}

https://www.cairn.info/revue-sociologies-pratiques-2008-2-page-81.htm

Distribution électronique Cairn.info pour Presses de Sciences Po.

(C) Presses de Sciences Po. Tous droits réservés pour tous pays.

La reproduction ou représentation de cet article, notamment par photocopie, n'est autorisée que dans les limites des conditions générales d'utilisation du site ou, le cas échéant, des conditions générales de la licence souscrite par votre établissement. Toute autre reproduction ou représentation, en tout ou partie, sous quelque forme et de quelque manière que ce soit, est interdite sauf accord préalable et écrit de l'éditeur, en dehors des cas prévus par la législation en vigueur en France. Il est précisé que son stockage dans une base de données est également interdit. 


\section{À chacun son psy. La diffusion des pratiques psychologiques en prison}

Corinne Rostaing ${ }^{1}$

Les dispositifs "psy " ont investi depuis longtemps la prise en charge des détenus dans les prisons. Plus récemment ces dispositifs se sont développés auprès des personnels pénitentiaires. L'article analyse la diffusion de ces pratiques; ce qu'elles apportent aux personnels dans un contexte de forte pénibilité, et les risques qu'elles portent si elles constituent l'unique réponse de l'institution à ces conditions de travail.

Les prisons sont le reflet de ce qui se passe dans notre société et, à ce titre, connaissent des formes de psychologisation des rapports sociaux de travail $^{2}$, qui se traduisent par une progression au sein du monde carcéral des manières psychologisantes d'agir sur autrui, liée au développement continu de services et de dispositifs « psy » en prison, à leur décloisonnement et à leur extension du côté des personnels. Comment ces processus contribuent-ils à faire évoluer, à la marge ou davantage, l'institution carcérale qui apparaît souvent, pour le sens commun, comme immobile?

Cet article entend suivre la progression de ces pratiques psychologiques : comment s'est-elle opérée ? A-t-elle des liens avec la prise en charge psychiatrique des détenus ? Quelles formes adopte-t-elle ? Quels en sont les enjeux et les effets réels ? Si ce processus apporte des outils relationnels et réflexifs aux personnels, s'il marque pour l'institution une prise en compte récente des difficultés au travail, il présente aussi des limites et des risques, notamment une tendance à l'individualisation des problèmes.

Cette analyse s'appuie sur des observations de la vie carcérale, des résultats de questionnaires et des entretiens avec des détenus, des soi-

1. Université Lumière de Lyon II - MoDys (UMR CNRS 5264).

2. Cette notion, relativement ancienne, concerne plus largement l'ensemble des rapports sociaux. Voir notamment les travaux de N. Elias (1975). Lire aussi L. Demailly (2006). 
gnants (psychiatres, psychologues, infirmiers) et des personnels de surveillance réalisés lors d'une recherche sur la violence en prison, menée dans cinq établissements (Chauvenet, Rostaing, Orlic, 2008). Elle s'intéresse tout d'abord au recours aux «psys » (psychiatres, psychologues, infirmiers psychiatriques) auprès des détenus, avant d'analyser en deuxième partie le mouvement d'extension de ce type d'interprétations vers les personnels de surveillance et leurs situations de travail.

\section{Du côté des détenus, la place toujours croissante des « psys »}

La place importante des psychiatres et psychologues auprès des détenus est ancienne, prison et psychiatrie ayant établi des liens depuis longtemps ${ }^{3}$. Leur entrée en prison a été institutionnalisée par la réforme Amor de $1945^{4}$ avec la création des centres médico-psychologiques et leurs statuts ont été étendus par la réforme des Services médico-psychiatriques régionaux (SMPR) en $1977^{5}$. Leur place s'est encore accentuée ces dernières années avec l'incarcération d'un nombre croissant de personnes atteintes de troubles psychiatriques et la prise en charge «psy » d'une partie grandissante des détenus.

\section{La prise en charge carcérale de personnes atteintes de troubles psychiatriques}

L'article 64 du Code pénal de 1810 qui reconnaît l'irresponsabilité en cas de démence devait marquer, avec la création de l'asile par la loi du 30 juin 1838, une séparation radicale entre l'hôpital psychiatrique et la prison, en permettant l'internement à l'asile des personnes jugées irresponsables de leurs délits ou crimes. Les deux «institutions totales » (Goffman, 1968) ont pourtant été longtemps associées, parce qu'elles symbolisaient des lieux de ségrégation et qu'elles s'occupent, encore aujourd'hui, de publics proches.

3. Notamment par les travaux criminologiques et les liens cliniques et institutionnels entre la médecine légale et la psychiatrie. Lire l'article de J. Ayme in Dormoy (1995: 27-37).

4. Pour une mise en perspective historique, voir Bruno Milly (2001 : 79-104).

5 . La circulaire de 1977 «libère » les médecins psychiatres de leur subordination au chef d'établissement pénitentiaire. 
Ces dernières années, les prisons accueillent de plus en plus de personnes atteintes de troubles psychiatriques. Le remplacement de l'article 64 de 1810 par l'article 122-1 du Code pénal de 1992 laisse en effet survivre une responsabilité pénale en cas d'altération des facultés mentales (« la personne demeure punissable »). Dans ce cas, le trouble mental constitue «seulement» une circonstance permettant la modulation de la peine. Seule l'abolition des facultés mentales constitue un motif de non-responsabilité pénale mais ce motif est moins souvent retenu, peut-être du fait de l'ouverture de l'hôpital psychiatrique sur la cité (politique de secteur, réduction du nombre des quartiers fermés, durée d'hospitalisation plus courte).

Aussi la prison est-elle devenue peu à peu le lieu d'enfermement des personnes ayant des troubles psychiatriques qui autrefois auraient été placées en hôpital psychiatrique. La prise en charge carcérale de cette population pose de nouvelles interrogations sur la mission de la prison, mettant au jour les contradictions entre le système judiciaire qui considère les personnes comme responsables de leurs actes et l'institution carcérale qui s'avère dans l'incapacité de les « soigner et / ou punir » (Dormoy, 1995 : 11-17).

\section{La prise en charge "psy » de détenus de plus en plus nombreux}

Une partie croissante de la population carcérale a besoin ou fait l'objet d'une prise en charge «psy ». Cela s'explique par la transformation des caractéristiques sociodémographiques de la population incarcérée, notamment le nombre croissant d'entrants ayant des antécédents psychiatriques. L'incarcération produit ou amplifie les perturbations psychologiques. L'isolement affectif, la non-acceptation de la peine, sa longueur, la promiscuité, l'inactivité aggravent les risques d'automutilations et de suicides. L'amplification des troubles a conduit, dans plusieurs situations rencontrées, au transfert en hôpital psychiatrique ou en Unité pour malades difficiles.

Le déploiement de cinq dispositifs favorise également le suivi d'une population toujours plus nombreuse :

- le dépistage systématique des troubles psychiques au moyen d'un entretien d'accueil avec les entrants contribue à augmenter la proportion de la population incarcérée suivie : ainsi, $52 \%$ des entretiens réalisés dans les établissements dotés d'un SMPR ont donné lieu à une préconi- 
sation de suivi psy contre $20 \%$ des entretiens réalisés dans les autres établissements (Coldefy, $2005: 5$ ).

- La mise en œuvre de protocoles de prévention du suicide favorise aussi le repérage des détenus «à tendance suicidaire » qui sont alors invités à rencontrer un psychologue (Golovine, 2004 : 191).

- La fréquence des conduites addictives au sein de la population carcérale (54\% des entrants en détention en 2003 déclarent consommer au moins une substance psycho-active : psychotropes, alcool, drogues illicites) accroît le taux de recours aux soins psychiatriques qui est près de dix fois supérieur à celui observé pour l'ensemble de la population (Coldefy, $2005: 5$ ). Les « antennes toxicomanie » visent ainsi à accueillir des toxicomanes et à leur proposer des traitements de substitution.

- La création du suivi sociojudiciaire avec injonction de soins, par la loi du 17 juin 1998, «incite» les auteurs d'infractions sexuelles, qui représentent aujourd'hui un quart des détenus, à commencer un traitement en détention. En cas de refus de soin, ils ne pourront obtenir aucune remise de peine supplémentaire.

- La mise en place depuis 2000 du Projet d'exécution des peines est une autre occasion, pour les condamnés à de longues peines, de s'entretenir avec un psychologue afin de définir les objectifs prioritaires (formation, travail, soins médicaux, paiement des parties civiles) à poursuivre au cours de leur peine.

Pour toutes ces raisons, le nombre de détenus concernés par une prise en charge psy ne cesse d'augmenter. La moitié des détenus d'une maison centrale étudiée était ainsi concernée par un soutien psy. Cette prise en charge prend des formes différentes : de l'entretien aux soins courants sous forme de consultations et de traitement médicamenteux, de la psychothérapie individuelle, souvent verbale, aux thérapies collectives (créative, théâtrale, par la danse, par le jeu), jusqu'à l'hospitalisation dans le cadre du SMPR.

\section{Du côté des personnels.}

\section{Le processus de psychologisation des rapports sociaux}

Ce processus s'est développé selon deux modalités, d'un côté la multiplication des interactions professionnelles entre personnels pénitentiaires et soignants à propos des détenus, de l'autre l'entrée de psychologues au service spécifique des personnels pénitentiaires, essentiellement des 
surveillants et des travailleurs sociaux ${ }^{6}$. Autant le travail des psys auprès des détenus constitue un phénomène ancien et largement traité ${ }^{7}$, autant l'entrée des psychologues auprès des personnels est récente et peu médiatisée ${ }^{8}$.

\section{La multiplication des interactions entre personnels de surveillance et soignants}

La réforme de janvier 1994 qui donne la responsabilité et le contrôle de l'offre de soins en prison à l'administration de la santé a permis de définir plus clairement les rôles et les obligations des services médico-psychiatriques et ceux de l'administration pénitentiaire. Et une politique de décloisonnement a contribué à développer les relations entre les membres de ces différents services et à faciliter les collaborations interprofessionnelles, notamment pour la prévention des suicides. Cette politique s'est traduite par la mise en place de commissions pluridisciplinaires comme la commission d'affectation des détenus en cellule et la commission de classement pour l'attribution des postes de formation ou de travail qui valorisent une approche interdisciplinaire, plus complète, du détenu :

L'observation de la commission d'attribution des postes de travail au sein d'un centre de détention montre que, pour neuf des onze cas discutés, sont évoquées des références à l'état de santé du détenu, essentiellement psychique, comme le montre cet exemple : la chef du bâtiment parle de la demande de travail de monsieur B. qui «fait du forcing pour travailler». La surveillante le décrit comme « un détenu difficile, un peu bizarre». Les deux infirmiers psy sont favorables à son classement, relatant les progrès de M. B., sa prise régulière de médicaments ; l'infirmière psy estime que «travailler pour lui, c'est plutôt thérapeutique car il est un peu dépressif ». La conseillère d'insertion s'interroge sur son instabilité, les chefs soulignent ses difficultés relationnelles avec ses codétenus. Le contremaître ne souhaite pas son classement à l'atelier du fait de la présence d'outils. Tenant compte de ces avis, la chef propose de classer M. B. à la buanderie, ce qui est validé par la commission.

6. Les personnels les plus concernés sont ceux qui sont en contact direct avec les détenus. Au cours de notre enquête, nous n'avons pas rencontré de personnels administratifs ayant fait appel au psychologue.

7. Parmi eux, Dormoy (1995), Psychologues et psychologies (2001), Bellemin-Noël (2003), Golovine (2004), Loiseau (2007).

8. Un seul article trouvé concerne le travail spécifique de psychologues auprès des personnels, celui de C. Bellemin-Noël, V. Geismar et H. Pecqueur (2003). 
Les formations, souvent «maison », assurées par des soignants, favorisent également les interactions avec les personnels de surveillance. Elles visent à aider les surveillants dans leurs relations avec les détenus (comme le montrent les intitulés suivants : «savoir gérer la relation», « la relation d'aide») ou dans leurs rapports au travail (comme «la gestion du stress »). Près de la moitié des SMPR dit avoir formé des professionnels pénitentiaires (Coldefy, 2005 : 9). L'animation, par des psychologues, de groupes de réflexion sur différents thèmes (addictions, agressions, suicides...) complète la connaissance des personnels sur la population carcérale et encourage leur réflexivité sur leurs pratiques.

Ces échanges ne sont toutefois pas toujours aussi développés dans chaque établissement ou entre tous les membres des services. Les surveillants se plaignent souvent de ne pas être suffisamment informés de l'état de dangerosité que peuvent présenter certains détenus et dénoncent les suspensions de peine de quartier disciplinaire par les psychiatres.

\section{À chacun son " psy " : l'entrée des psychologues des coursives}

Dès le début des années 1990, l'administration pénitentiaire a mis en place une politique de recrutement de psychologues des coursives, dont la mission à l'origine était le « soutien technique » aux personnels. Mais son application n'a pas toujours été simple :

«Globalement, quand je suis arrivée sur ce poste, il était à pourvoir depuis huit mois. Le directeur était défensif, c'est difficile de reconnaître que son personnel peut aller mal. Il y a aussi ici une culture du secret, on ne dit rien, secret professionnel, cela ne doit pas sortir des murs. Il lui a fallu des avis positifs pour comprendre que les personnels, eux aussi, avaient besoin d'une psychologue !»

(Psychologue clinicienne, en poste depuis deux ans)

Le terme de «psychologue des coursives » vise à différencier les «psys » (psychiatres, psychologues, infirmiers psychiatriques) s'occupant des détenus des psychologues réservés aux personnels. Il renvoie à leur présence sur le lieu même du travail, sur une coursive, dans un « rond-point », dans les couloirs, ce qui favorise leurs rencontres avec les surveillants. Dans trois des cinq établissements étudiés au cours de notre enquête, ces psychologues, uniquement des femmes, étaient facilement accessibles et leur présence contribue largement à leur légitimité : «La seule position acceptable pour nous faire connaître et reconnaître 
consiste alors en une sorte d' "être là", ne relevant pas de l'agir, mais d'une position subjective de "non-faire" »(Bellemin-Noël, Geismar, Pecqueur, 2003 : 47).

Quel rôle jouent ces psychologues du personnel? «Ni expert ni thérapeute, le psychologue propose une présence attentive, soucieuse de la vie psychique de celui qui s'adresse à lui »(Bellemin-Noël, Geismar, Pecqueur, 2003 : 48). Leur prise en charge vise à limiter l'usure au travail, particulièrement fréquente dans ce métier (Chauvenet, Rostaing, Orlic, $2008: 55)^{9}$. Un médecin du travail rencontré en maison centrale décrit quelques symptômes de cette usure :

«Depuis 1993, je vois tous les agents, certains deux, trois ou quatre fois par an. Tout le monde ici a besoin de tabac et d'alcool pour tenir. On a beaucoup de cas d'alcoolisme. Il y a une tension nerveuse. Ce n'est pas sain de surveiller quelqu'un. Ils ont l'impression d'être détenus. Beaucoup n'arrivent pas le matin à entrer au boulot. Ce n'est pas un travail créatif et valorisant. Il y a beaucoup de dépressions en pénitentiaire. Il y a eu une vague de suicides : quatre suicides dans mon secteur, et un cas de maladie dépressive grave. »

L'usure au travail ${ }^{10}$ liée aux conditions de travail intensifiées (manque de personnel, accroissement des demandes de la part des détenus, conditions difficiles de travail) se traduit par la peur de se rendre en détention, par le fait de ne plus supporter le contact des détenus, voire les tensions avec la hiérarchie, par des comportements violents au travail ou dans leur vie privée :

«Certains agents ne se sont pas reconnus, ils n'avaient plus de barrière, ils ont insulté la personne. Il faut les aider à réfléchir, c'est-à-dire se demander pourquoi il a été obligé de se mettre en colère ; est-ce la goutte qui a fait déborder le vase ? Est-ce qu'il divorce, est-ce qu'il est fatigué ? Il y a l'usure professionnelle, certains ont des personnalités fragiles et leur travail exacerbe ce qui n'allait pas au départ. Il y a trop de travail, il manque trop de personnel... »

(Psychologue, en centre de détention depuis trois ans)

L'aide psychologique constitue également un soutien après un événement traumatisant (prise d'otage, agressions, mutinerie, incendie, etc.)

9. Lorsqu'on demande aux surveillants de choisir l'aspect de leur travail qui leur paraît le plus difficile ( $\mathrm{N}=496$ réponses), l'usure au travail vient largement en tête des réponses dans les cinq établissements, devant l'insécurité du métier et l'image du métier.

10. Le stress au travail, l'usure, le burn-out des personnels ont été étudiés notamment par M. Loriol (2000) ; M. Delbrouck (2003) ; M. Buscatto, M. Loriol, J.-M. Weller (2008). 
que près de $60 \%$ des surveillants interrogés disent avoir vécu depuis leur recrutement (plus spécifiquement, $45 \%$ disent avoir été directement confrontés à un suicide) (Chauvenet, Rostaing, Orlic, 2008 : 154). Le travail de «debriefing » proposé vise à faciliter le retour au travail des personnes fragilisées. Ce soutien ${ }^{11}$ vient en quatrième position, loin derrière celui des collègues de travail (71 \% des répondants), des proches (62\% des répondants) et des gradés (un quart des répondants) : $18 \%$ des répondants concernés disent avoir trouvé un soutien auprès du psychologue du personnel ${ }^{12}$.

Face à une définition peu précise de ses missions, chaque psychologue propose une interprétation personnalisée de son poste, en fonction de son temps de présence et selon le nombre et la nature des demandes. Certaines traitent l'ensemble des difficultés des personnels et assurent un suivi thérapeutique, d'autres ont une vision plus restrictive de leur mission :

« Je travaille six heures par semaine en vacations. [...] On aborde les difficultés liées à leur profession, je réoriente quand ils me parlent de difficultés plus personnelles. Cela s'imbrique certes mais nous n'avons pas le temps! Faut définir son propre cadre. Je ne propose pas de suivi thérapeutique, cela n'est pas prévu dans la définition de mon poste, je suis d'abord une psychologue de coursives. Quand c'est trop compliqué, trop personnel, on réoriente. »

L'entretien individuel est le mode majoritaire. Des psychologues initient aussi des groupes de parole, souvent avec difficulté :

«Je leur ai proposé un groupe de parole entre surveillants mais en fin de compte, ça n'a pas marché. C'est vécu comme dangereux de dire ce qu'on a vécu, les situations difficiles, qu'on n'a pas su gérer... Il y a la peur d'être perçu comme un mauvais agent, peur d'être mis en échec.»

Leur offre de parole et d'écoute est appréciée par ceux qui l'ont sollicitée mais des réticences existent.

\section{Les enjeux et processus de psychologisation}

Si ce processus a des effets particulièrement positifs au sein d'une institution qui jusqu'à présent tendait à sous-estimer ou à nier l'existence

11. $\mathrm{N}=190$ répondants, plusieurs réponses possibles.

12. $12 \%$ déclarent également avoir trouvé un soutien auprès d'un médecin ou «psy » privé. 
de souffrances au travail (C. Dejours, avec J. de Bandt et C. Dubar, 1995), on peut cependant s'interroger sur ses enjeux, tant pour l'institution que pour les personnels, et sur le risque d'une individualisation excessive des problèmes.

\section{Une nouvelle politique institutionnelle vis-à-vis des surveillants ?}

Le recrutement de psychologues du personnel, qui existe déjà dans la police ou dans l'éducation, peut surprendre dans une institution peu habituée à ces pratiques. Les difficultés de recrutement des surveillants, les nombreuses démissions, les incapacités de travail, les arrêts de travail prolongés ou répétés ont « obligé » l'administration pénitentiaire à prendre en compte l'image dévalorisée de la profession et les difficultés spécifiques du travail en prison (la garde de personnes contre leur gré, la confrontation à la souffrance, le manque récurrent de moyens, une surpopulation accrue...). L'administration entend ainsi démontrer sa volonté d'une politique tournée vers ses personnels alors même que le manque d'effectifs humains face à la surpopulation carcérale la contraint à solliciter davantage ses surveillants.

Notre enquête a constaté une préoccupation plus forte de l'institution carcérale pour l'intégrité physique et psychique de ses personnels, qui se traduit par la protocolisation du suivi du personnel agressé (appel téléphonique à la victime, visite à l'hôpital, soutien psychologique proposé...) et par des situations de « debriefing » après des incidents en détention.

Le dispositif actuel est-il suffisant pour contrecarrer les problèmes de maintien en poste de ces personnels et leurs difficultés professionnelles ? Il reste à un niveau expérimental et l'offre est limitée : tous les établissements ne proposent pas de soutiens psychologiques et les formations ne concernent qu'une infime partie des personnels ; la durée réduite de la présence des psychologues limite l'écoute à la dimension professionnelle ou ne leur permet pas d'engager un réel suivi thérapeutique.

La pertinence de ce dispositif peut aussi être interrogée du fait de la marginalisation relative des psychologues du personnel. Ils travaillent relativement peu en coordination avec le médecin du travail si ce n'est pour donner suite à des signalements. Leurs relations avec les équipes médico-psychiatriques auprès des détenus sont faiblement instituées, ce qui est en partie lié aux missions des psychologues, à leur temps de présence limité et à leur espace de travail (entre la détention et des 
bureaux situés dans des bâtiments administratifs), fort éloigné des Unités de consultations et SMPR.

\section{Pour les personnels, entre apports et réticences}

La majorité des surveillants a le sentiment que les formations, les entretiens psychologiques et les interactions avec des soignants contribuent à renforcer leur professionnalité en complétant leur formation, jugée trop courte. Nous avons observé ces dernières années l'amélioration de la compétence relationnelle des surveillants avec les détenus, ce qui est une source de satisfaction pour les surveillants. La prise en compte de la dimension psychique des détenus a sans doute contribué à établir des relations mieux adaptées à chacun, à calmer le jeu en parlant doucement à un détenu énervé, à mettre en place une relation «plus pacifiée ». Les interventions à plusieurs, quand le détenu est en crise ou dans le refus total, sont mieux organisées (répartition des rôles, mise en place de parades pour «faire impression ») afin de montrer sa force tout en évitant d'y avoir recours. En enrichissant leur répertoire d'action, ils ont contribué à développer leur réflexivité sur leurs pratiques.

Malgré cela, existent des formes de résistance des personnels qui refusent d'aller voir un psychologue, du fait de leur non-familiarité avec l'approche psychologique mais également du fait de la féminisation de cette profession. Ces personnels majoritairement masculins sont moins enclins à se confier à des femmes et ils élaborent de multiples résistances à cette présence féminine dans un univers majoritairement masculin ${ }^{13}$. Il y a aussi le refus de certains personnels d'aller voir un psychologue sur leur lieu de travail. Les psychologues font part de la stratégie défensive des surveillants face au caractère jugé «intrusif » de leur présence, soit par le refus d'aller les voir ("Ils ne viennent pas jusqu'à mon bureau, même quand ils vont très mal»), soit même par l'acceptation de rencontres informelles, devant un café, dans un couloir qui limitera de fait la profondeur des entretiens. Des surveillants doutent de l'efficacité de cette aide psychologique qui ne constitue, selon eux, qu'un soutien ponctuel.

Le recrutement institutionnel des psychologues incite certains surveillants à préférer une aide psychologique extérieure, à la fois pour ne pas

13. Processus analogue à l'entrée des surveillantes dans les détentions masculines in G. Malochet (2005). 
en informer la hiérarchie ou leurs collègues ("Ils n’ont pas à savoir que je vais voir quelqu'un »), préserver la confidentialité de leurs propos et être libre d'aborder des questions plus personnelles. Malgré l'insistance de ces psychologues à souligner leur indépendance par rapport à l'institution, il arrive qu'un membre du personnel fasse l'objet d'un « signalement» par un supérieur hiérarchique ou par le médecin du travail. Cette situation interroge quant au secret médical et à ses limites ${ }^{14}$ et quant aux pressions qui peuvent s'exercer sur des personnels « invités » à reconnaître la nécessité d'être soignés, au risque d'être mal perçus par leur hiérarchie.

\section{Le risque d'une surinterprétation " psy » des problèmes des détenus}

Par ailleurs, le développement des suivis «psy » des détenus conduit des surveillants à surestimer la présence de détenus « à problèmes psychiques » et se traduit par la peur des détenus jugés imprévisibles. Le caractère « imprévisible » de la prison est le premier qualificatif retenu par $88 \%$ des surveillants pour décrire le climat de la détention (Chauvenet, Rostaing, Orlic, 2008 : 52). Près de la moitié des surveillants rencontrés qui disent avoir été agressés l'imputent à l'état mental de leur agresseur ${ }^{15}$. La question de la violence tend à être de plus en plus «psychiatrisée » alors que d'autres éléments comme l'enfermement, pour une durée de plus en plus longue, la cohabitation avec des personnes non choisies, l'incertitude, la peur, la provocation d'un surveillant permettraient de comprendre (au sens de Weber) ce comportement, celui d'une personne normale soumise à des conditions extrêmes. Des psychiatres, tout en reconnaissant une augmentation du nombre des personnes ayant des troubles «psy », considèrent qu'il y a une tendance à surestimer leur place :

«On nous donne de plus en plus d'importance même si on n'a pas plus de pouvoir. Ici tout est psychiatrisé, tout problème d'agression est une question psychiatrique, or c'est souvent un problème entre le surveillant qui a du mal à gérer et le détenu qui ne supporte pas un refus », dit ce psychiatre du centre de détention.

14. Des échanges informels entre le médecin du travail ou une psychologue et la direction (auxquels nous avons assisté) montrent une porosité des informations partagées à propos du divorce d'une surveillante, de l'alcoolisme d'un surveillant ou encore de la dépression d'un gradé.

15. Contre un tiers des agressions selon M. Guillonneau, A. Kensey (1998: 49). 


\section{Le risque d'une individualisation des problèmes des surveillants}

Le dispositif de soutien psychologique des personnels, valorisant une manière psychologisante d'agir sur autrui, interroge quant au déplacement actuel des questions institutionnelles ou politiques à un niveau individuel. Il constitue une réponse peu onéreuse pour une institution qui dispose de faibles moyens. L'offre d'une aide personnalisée permet à la fois d'apaiser le malaise de certains surveillants quant à leurs missions ou à leur travail et de reconnaître les difficultés spécifiques de leur profession. Les entretiens avec un(e) psychologue constituent des espaces de parole et de réflexivité qui aident les surveillants à « tenir », à redonner un sens positif à leur travail ou à consolider les plus fragilisés.

Si le soutien psychologique des personnels en cas d'incidents graves ou violents constitue un réconfort incontestable et une forme de reconnaissance de la souffrance de la victime, cette réponse semble insuffisante face à l'intensification du rythme du travail et à l'usure professionnelle qui en découle. Elle ne traite pas une question de fond, celle de la dégradation des conditions de vie et de travail liées à la surpopulation grandissante du fait de la politique sécuritaire. Ces questions essentielles sont renvoyées par l'administration à un niveau individuel : la fragilité voire l'incapacité de certains membres du personnel à supporter le rythme du travail ou les tensions.

\section{Conclusion}

Cet article a exploré les formes, les enjeux et les limites du processus récent de psychologisation des rapports sociaux de travail en prison. Il s'est d'abord intéressé à l'important dispositif psychiatrique et psychologique auprès des détenus puis à l'extension de ce type d'interprétations vers les personnels de surveillance, par le biais de formations assurées par des soignants et par le développement d'une politique de décloisonnement entre services sociaux, médico-psychiatriques et pénitentiaires.

Ce dispositif a été consolidé par le recrutement de psychologues du personnel, ce qui constitue une perspective novatrice pour une administration pénitentiaire peu habituée à de telles pratiques. Il a démontré la volonté de cette administration de reconnaître certaines difficultés de 
recrutement et de souffrances de ses personnels. L'extension des pratiques psychologiques à l'ensemble des acteurs concourt à rapprocher les pratiques de l'institution carcérale de celles des autres institutions. La présence de ces personnels, extérieurs à l'administration pénitentiaire, essentiellement féminins, favorise de nouvelles pratiques professionnelles. La diffusion de pratiques psychologiques donne aux personnels des outils originaux pour analyser leurs actions et ce processus est à rapprocher du processus de judiciarisation de la prison (Rostaing, 2007), le droit devenant une ressource pour les personnels comme pour les détenus. La diffusion de pratiques psychologiques pourrait-elle contribuer à une transformation en profondeur de l'institution carcérale ?

La diffusion des pratiques psychologiques aux personnels marque une évolution, une évolution à la marge de l'institution carcérale. L'extension actuelle de ce processus présente en effet des limites du fait de réticences des personnels de surveillance. Cette offre de services personnalisés comporte également le risque d'une individualisation excessive des réponses à des questions fondamentales, notamment sur le sens de la peine pour les détenus ou les missions des surveillants, et actuellement sur les moyens humains et matériels permettant de freiner l'intensification du travail et les pressions accrues sur les personnels du fait d'une surpopulation carcérale toujours croissante.

Corinne.Rostaing@univ-lyon2.fr 


\section{Références bibliographiques}

Bellemin-Noël C., Geismar V. et Pecqueur H. (2003), «Psychologues auprès des personnels pénitentiaires », Journal des psychologues, $n^{\circ} 210$, p. 46-49 (un dossier complet est consacré au «psychologue en milieu carcéral » dans ce Journal des psychologues).

Buscatto M., LoRIOl M., WelleR J.-M. (2008), Au-delà du stress au travail. Une sociologie des agents publics au contact des usagers, Ramonville, Erès.

Chauvenet A., Rostaing C., Orlic F. (2008), La violence carcérale en question, Paris, PUF, « Le Lien social ».

ColdeFY M. (2005), « La prise en charge de la santé mentale en 2003 », Études et Résultats, $\mathrm{n}^{\circ} 427$.

Dejours C. (1995), La France malade du travail (avec J. de Bandt et C. Dubar), Paris, Bayard.

DelBrouck M. (2003), Le burn-out des soignants, Bruxelles, De Boeck, 2003.

Demailly L. (2006), " La "psychologisation" des rapports sociaux comme thématique sociologique ", in Bresson M., La psychologisation de l'intervention sociale, Paris, L'Harmattan.

Dormoy O. (1995), Soigner et/ou punir, Paris, L'Harmattan.

EliAS N. (1975), La dynamique de l'Occident, Paris, Calmann-Lévy.

Goffman E. (1968), Asiles. Étude sur la condition sociale des malades mentaux, Paris, Éditions de Minuit.

Golovine P. (2004), « Un psychologue en prison : entre logique psychiatrique et logique judiciaire ", Psychotropes, volume 10, $\mathrm{n}^{\circ}$ 3-4.

GUILLONNEAU M., KENSEY A. (1998), «Les à-coups. Étude statistique des agressions contre le personnel de surveillance à partir de 376 rapports d'incident ", D.A.P., Travaux \& Documents, 53.

LOISEAU N. (2007), «Le psychologue clinicien et la prison », in Schauder S., Pratiquer la psychologie clinique, Paris, Dunod, p. 227-257.

LORIOL M. (2000), Le temps de la fatigue. La gestion du mal-être au travail, Paris, Anthropos.

MALOCHET G. (2005), « Dans l'ombre des hommes. La féminisation du personnel de surveillance des prisons pour hommes ", Sociétés contemporaines, 59-60, p. $199-220$.

MiLly B. (2001), Soigner en prison, Paris, PUF, « Sociologies ».

«Psy dans la prison » (2001), in Psychologues et psychologies, $\mathrm{n}^{\circ} 157$.

RostaING C. (2007), "Processus de judiciarisation carcérale : le droit en prison, une ressource pour les acteurs ? », Revue Droit et Société, n 67, p. 577-595. 